the developmental increase in SSC usually present in childhood is disrupted in autistic children. Previous studies in animals, cited by the authors, have shown that changes in brain serotonin during development may affect synaptic connections in the sensory cortex and alter neuronal differentiation.

\title{
MPH RESPONSE PREDICTORS IN ATTENTION DEFICIT DISORDERS
}

Response to methylphenidate (MPH) in 36 boys (7-12 years) with attention deficit hyperactivity disorder (ADHD) was examined in a double-blind, placebocontrolled, crossover study at the National Centre for Child and Adolescent Psychiatry, Oslo, Norway. Significant improvements in the level of hyperactivity at home or at school and in conduct problems at school were obtained in response to $\mathrm{MPH}(0.5 \mathrm{mg} / \mathrm{kg} /$ day) in $83 \%$ (behavior was at normal levels in $60 \%$ ). Neuropsychological tests showed MPH-induced improvements in sustained attention (Children's Checking Task [CCT] and Continuous Performance Test [CPT] of vigilance), working memory (Paced Auditory Serial-Addition Task [PASAT]) and motor coordination (Maze test).

Pre-treatment high levels of hyperactivity and inattention, relatively young age, and absence of emotional disorders were correlated significantly with normalization of hyperactive behavior in $71 \%$ of cases. Test performance and neurodevelopment scores had no predictive value. (Zeiner P, Bryhn G, Bjercke C, Truyen K, Strand G. Response to methylphenidate in boys with attention-deficit hyperactivity disorder. Acta Paediatr March 1999;88:298-303). (Respond: P Zeiner, National Centre for Child and Adolescent Psychiatry, PO Box 26, Vinderen, 0319 Oslo, Norway).

COMMENT. The degree of hyperactivity in children with ADHD is correlated with response to MPH treatment. This finding in the Oslo study corroborates previous reports, both in clinical and animal studies, showing that subjects with the highest levels of motor activity are most likely to respond positively to MPH, whereas those with lower activity levels are sometimes made worse (Millichap et al. Am I Dis Child 1968;116;235-244; Ann N Y Acad Sci 1973;205:321-334). In these studies, neuropsychological test performances were also improved, and the occurrence of subtle signs of neurological abnormality was a likely predictor of response to MPH. Taylor et al (Psychol Med 1987;17:121-143) have also reported that high levels of motor activity, impaired attention and motor coordination, younger age and absence of emotional problems may predict a response to MPH.

Denney CB and Rapport MD, University of Hawaii, evaluated the validity of models designed to predict MPH-responsiveness in children with ADHD (I Am Acad Child Adolesc Psychiatry April 1999;38:393-401). They concluded that the magnitude of reponse to MPH measured by classroom attention and behavioral inhibition are correlated and predictive of academic performance and teacherrated behavior.

Comorbid anxiety and ADHD response to MPH. Diamond IR, Tannock R, and Schachar RJ, University of Toronto, examined the effect of comorbid anxiety on response to MPH in ADHD ( $L$ Am Acad Child Adolesc Psychiatry April 1999;38:402-409). Contrary to previous reports, behavioral response and side effects with MPH were unaffected by comorbid anxiety after 4 months of treatment, titrated to a dose of $0.7 \mathrm{mg} / \mathrm{kg}$. Difficulties in measurement of childhood anxiety may account in part for differences in results.

\section{BEHAVIORAL PROBLEMS IN LEARNING DISABLED CHILDREN}

The relationships between learning difficulties (LD) and behavior problems (BP) in 11 to 12-year-old children was determined at the Royal 
Children's Hospital, Melbourne, Australia. Of children identified with LD, more than half had BP diagnosed by DSM-III-R criteria. Those with mathematics difficulties were at greater risk of BP than children who had spelling problems (69\% cf $31 \%$ affected). Children with a single LD (spelling or math) were equally likely to have an internalizing diagnosis (anxious-fearful), while those with both types of LD were more prone to externalizing disorders (hostile-aggressive or hyperactive-distractible). Of children identified with LD at 7 to 8 years, $80 \%$ had persisting literacy difficulties and $57 \%$ had arithmetic problems at 12 years of age. (Prior M, Smart D, Sanson A, Oberklaid F. Relationships between learning difficulties and psychological problems in preadolescent children from a longitudinal sample. I Am Acad Child Adolesc Psychiatry April 1999;38:429-436). (Respond: Professor Margot Prior PhD, Department of Psychology, Royal Children's Hospital, Parkville, Victoria, Australia 3052).

COMMENT. Children with learning disorders are at high risk of developing behavior problems, and children with LDs and BPs have persistence of difficulties through grade school years, particularly for boys. Mathematical LD predisposes to anxiety disorders whereas multiple LDs are predictive of hyperactivity or hostileaggressive behavior.

Cocaine exposure and behavioral problems. Children of drug abusers (CDAs) are at increased risk of internalizing and externalizing problems, attention deficits, and impulsivity. Stanger $C$ and associates, University of Vermont, found that CDAs showed more behavior problems than matched nonreferred children, but significantly less psychopathology than a sample of children referred for mental health services (I Am Acad Child Adolesc Psychiatry April 1999;38:421-428).

\section{CORTICAL DYSFUNCTION IN AUTISM}

Cerebellar and cortical systems in 26 high-functioning adolescents and young adults with autism were examined and compared to 26 healthy controls at the University of Pittsburgh School of Medicine. Reflexive, visually guided (cerebellar modulated) saccadic eye movements were normal whereas volitional (neocortical) saccade tasks (oculomotor delayed-response and antisaccade) were abnormal. The results demonstrate neocortical, not cerebellar, dysfunction in autism, suggesting deficits in higher cognitive mechanisms and impairments in spatial working memory and executive control over reflexive behavior. (Minshew NJ, Luna B, Sweeney JA. Oculomotor evidence for neocortical systems but not cerebellar dysfunction in autism. Neurology March 1999;52:917-922). (Reprints: Dr Nancy J Minshew, University of Pittsburgh School of Medicine, 3811 O'Hara St, 430 Bellefield Towers, Pittsburgh, PA 15213).

COMMENT. Both cerebellar and neocortical dysfunctions have been proposed as a neurologic basis for autism. Dysfunction of cerebellar vermal lobules VI and VII should be associated with dysmetric saccadic eye movements. In the present study, cerebellar-mediated saccades were normal but neocortical control of oculomotor eye movements was impaired. The findings support neuropsychological evidence of deficits in cortical, voluntary regulation of attentional focus in autism.

A new hypothesis for autism is proposed by DeLong GR, Duke University Medical Center (Neurology March 1999;52:911-916). Autism occurs in two forms: 1) Secondary to early infantile, bilateral brain damage (eg. epileptic medial temporal sclerosis, herpes simplex encephalitis, infantile spasms, tuberous 